\title{
Reformbedarf der
}

\section{Pflegeversicherung}

\section{JÜRGEN GOHDE}

Dr. h. c. Jürgen Gohde ist Vorstandsvorsitzender des Kuratoriums Deutsche Altershilfe (KDA) in Köln

Seit mehreren Jahren besteht bei der Reform der Pflege kein Erkenntnis- sondern ein Umsetzungsdefizit. Unser Land braucht einen Nationalen Aktionsplan „Teilhabe und Pflege“. Eine zukunftsfähige Infrastruktur der Pflege braucht bei knappen Ressourcen ein zielgenaues Gesamtkonzept für Teilhabe, Pflege und Betreuung im Quartier. Die Stärkung der Grundsätze «ambulant vor stationär", Prävention und Rehabilitation vor Pflege ist unerlässlich, wenn nicht personelle, finanzielle Engpässe und Versorgungslücken entstehen sollen. Gute Pflege nützt allen. Um sie zu fördern, sind Rahmenbedingungen für die verbindliche Kooperation der Akteure vor Ort (Kommunen und Sozialversicherungen, Unternehmen der Pflegebranche sowie der Zivilgesellschaft und der Angehörigen zu verbessern und eine gute Koordination der Leistungen vor Ort zu ermöglichen.

\section{Die Einführung der sozialen Pflegeversicherung als sozialpolitische Errungenschaft}

Um den heutigen Reformstau aufzulösen und Strategien zu entwickeln für eine den heutigen Wünschen und Bedürfnissen gerecht werdende Struktur moderne Versorgungs- und Beteiligungslandschaft lohnt es sich in die Zeit zurückzugehen, in der die ersten Impulse für eine solidarische Absicherung des Pflegerisikos gesetzt wurden.

„Bei der Analyse der bestehenden Situation der Altenhilfe und bei Überlegungen zur konzeptionellen Weiterentwicklung standen zwar zunächst Art, Qualität und Umfang der Leistungserbringung im Vordergrund, doch wurden die sozialpolitischen Rahmenbedingungen nicht außer Acht gelassen. Ein zentrales Thema der sozialpolitischen Diskussion zu Beginn der 70er Jahre bildete die Frage nach der begrifflichen Trennung von Behandlung und Pflege und der damit verbundenen organisatorischen Trennung nach entsprechenden Versorgungsstrukturen und Finanzierungsbedingungen. Solidarisch gesicherte Finanzierung durch die Krankenkassen bei Behandlungsbedürftigkeit stand der individuell zu sichernden Finanzierung bei Pflegebedürftigkeit gegenüber und da Einkommen und Vermögen des Einzelnen oft nicht ausreichten, die Kosten der Pflege ganz oder nur zu einem Teil aus eigenen Einkünften und Vermögen aufzubringen, musste durch die Kommunen zu finanzierende Sozialhilfe in Anspruch genommen werden. Eine 1973 ins Leben gerufene Arbeitsgruppe im KDA, die aus Fachleuten aus den Gebieten Geriatrie, Gerontopsychiatrie, Kommunalverwaltung und Sozialwissenschaft bestand, kam in ihrem 1974 veröffentlichten „Gutachten über die 
stationäre Behandlung von Krankheiten im Alter und über die Kostenübernahme durch die gesetzlichen Krankenkassen“ zu dem Schluss: „Die Behandlung von kranken alten Menschen in großen Pflegeabteilungen, Pflegeheimen oder Krankenheimen[...], fällt bei gesetzlich Krankenversicherten in die Zuständigkeit der gesetzlichen Krankenkassen.“ Die Veröffentlichung dieses Gutachtens bildet einen Meilenstein in der Geschichte des KDA und rief zahlreiche Reaktionen hervor, die von krasser Ablehnung durch die Krankenkassen über moderate Kritik an der Argumentation des Gutachtens bis zur grundsätzlichen Zustimmung bei Wohlfahrtsverbänden und Kommunen führte.

Die Veröffentlichung bildete ebenso einen Meilenstein in der dann fast zwanzig Jahre anhaltenden Diskussion um eine Pflegeversicherung. An weiteren Vorarbeiten, unter anderem Überlegungen einer Bund-Länder-Arbeitsgruppe beim Bundesfamilienministerium, war das KDA beratend beteiligt. Es wäre nun ein großes Missverständnis, wenn man zu der Auffassung gelangen würde, dass das KDA mit seinem Gutachten nur Partei ergriffen hätte für die Koalition der Sozialhilfeträger und der Heimträger, die die Notwendigkeit eines neuen Kostenträgers für die Pflege öffentlich vor allem dadurch begründete, dass die Pflegebedürftigkeitsbedingte Sozialhilfeabhängigkeit eines modernen Sozialstaats unwürdig sei. Denn das Gutachten des KDA betonte vielmehr die Notwendigkeit für einen Begriff der Behandlung für Pflegebedürftige, der rehabilitationsorientierte Pflege, Verabreichung von Medikamenten, Therapi-

\section{Das KDA kann mit Recht darauf verweisen, zu den wichtigsten Wegbereitern der gesetzliche Pflegeversicherung zu gehören.}

Recht darauf verweisen, zu den wichtigsten Wegbereitern für die dann mehr als 20 Jahre später geschaffene gesetzliche Pflegeversicherung. Das neue Gesetz hatte aber auch Schwächen, auf die das KDA immer wieder aufmerksam macht(e). Die Regierung sollte jetzt umgehend die gesetzliche Definition der Pflegebedürftigkeit erweitern. Der zeitliche Aufwand für die allgemeine Betreuung, Anleitung und Aufsicht bei gerontopsychiatrisch veränderten Menschen müsse bei der Begutachtung der Pflegebedürftigkeit berücksichtigt werden, lautete die Forderung der KDAKuratoren im Rahmen einer Vollversammlung am 06.11.1998. Zehn Jahre später erarbeitete unter Leitung des KDA-Vorsitzenden Dr. h. c. Jürgen Gohde ein Expertenbeirat im Auftrag der Bundesregierung einen neuen Pflegebedürftigkeitsbegriff zur Reform der Pflegeversicherung." (50 Jahre KDA, Gründung- Geschichte- GegenwartAusblick, 2012, 47f).

Die historische Reminiszenz gibt für die gegenwärtige Situation und die aktuelle Reformdiskussion wichtige Hinweise:

Der Anstoß zum Entwurf einer gesetzlichen, solidarischen Absicherung des Pflegerisikos steht im Zusammenhang der Aufgabe des Kuratoriums Deutsche Altershilfe, die Lebenslagen älterer Menschen realistisch wahrzunehmen und aufmerksam zu machen auf Defizite in der Versorgung. Bei seiner Gründung hatte der damalige Bundespräsiden Lübke unter den Stichworten Selbstbestimmung und Teilhabe, einen Impuls zur Modernisierung der Versorgung für Menschen im Alter und mit Pflegebedarf den gesellschaftlichen Auftrag formuliert, der in der praktischen Arbeit zu einer Fülle von Innovationen zur Folge hatte. Der internationale Vergleich zeigte, dass in den 80er Jahren in Deutschland noch nie so viele

en, Reaktivierung etc. unter medizinischer Verantwortung vorsah. Insofern war das Gutachten ein Anstoß für einen neuen Pflegebegriff und ein Plädoyer für die Verbesserung der Qualität in der Versorgung von Menschen mit Pflegebedarf.

Es war damals allzu deutlich, dass mit einer schnellen Neuregelung nicht zu rechnen war. Das KDA kann mit
Menschen in privaten Haushalten versorgt wurden wie zuvor, ohne diese ein ähnliches Maß an Entlastung erfahren zu lassen wie in Nachbarländern. Der Grundsatz „ambulant vor stationär galt auch damals, doch wurden die Pflegenden im Stich gelassen. Der Ausbau haushaltsnaher Dienstleistungen unterblieb. Dieser hätte wie der Vergleich z. B. mit Skandinavien zeigt, zum Erhalt der
Selbstständigkeit und zur Vermeidung von Pflegebedürftigkeit einen wirksamen Beitrag leisten können. Der Impuls zum Ausbau der Tages- und Kurzzeitpflege wurde gegeben, und schrittweise umgesetzt, ohne dass man sagen könnte, dass bis heute eine konsequente Ambulantisierung der Hilfen verfolgt worden und ein tragfähiges Versorgungssystem entstanden wäre. Dennoch sind durch das „Türöffnungskonzept für Menschen mit Demenz“, den Vorschlag zur Einrichtung von Demenzservicestellen in NRW, durch die Studien zur Heimunterbringung (1975), oder zur Wohnungsanpassung (1986), die Entwicklung des Wohngruppenkonzepts und später der Perspektiven für eine Zukunft der Pflege im Quartier, die Anstöße zur Qualität der Ausbildung und der Forschung in der Pflege, zur Qualität in der Pflege zahlreiche handlungsrelevante Impulse gesetzt worden.

Der Impuls zur Entwicklung der rechtlichen Leistungsansprüche, die dem Leitbild eines selbstbestimmten, möglichst selbstständigen Leben in der eigenen Häuslichkeit entsprechen, wurde im Kontext von veränderten Erwartungen und Notwendigkeiten, internationalen Erfahrungen in Spannung mit dem finanziell Machbaren konzipiert. Manche dieser Innovationen haben die Altenhilfe nachhaltig verändert, andere wesentliche Impulse bis heute unerledigt:

Dazu zählen nicht nur die bis heute nicht realisierte Einführung des neuen Pflegebedürftigkeitsbegriffs, die weit mehr als die im Pflege-Neuausrichtungsgesetz erreichte finanzielle Besserstellung von Menschen mit Einschränkungen ihrer Alltagskompetenz im Vorgriff auf eine gesetzliche Einführung des neuen Begriffs, die Gleichstellung von Menschen mit Pflegebedarf gefördert hätte, sondern vor allem auch die bereits im Gutachten von 1974 deutliche angesprochene Herausforderung einer „rehabilitationsorientierten Pflege“, die wie die Arbeit des KDA zeigt, in enger Beziehung steht zur Entwicklung von neuen sozialen Wohnformen im Sozialraum, der konsequenten Ambulantisierung von Leistungsformen, wie der Bewusstmachung der Potentiale der Zivilgesellschaft und die Förderung von Beteiligungsprozessen der Zivilgesellschaft.

Das hat freilich in der Vergangenheit nicht verhindert, dass auch das KDA 
häufig mit stationären Angeboten identifiziert worden ist und der Grundsatz „Ambulant vor stationär“, der einvernehmlich verfolgt werden soll, nur unzureichend realisiert ist. Diese Entwicklung ist zweifelsohne gefördert worden durch die im Zusammenhang der Medizinalisierung des Leistungsgeschehens der Pflegeversicherung nach 1994 zu beobachtende Maßnahmeorientierung und die Etablierung eines Pflegebedürftigkeitsbegriffs, der die Lebenslagen von Menschen mit Pflegebedarf -anders als es der fachwissenschaftliche Standard und die Bedürfnisse der Menschen nahelegen-, nicht ausreichend wahrnimmt, dadurch dass er den Schwerpunkt auf die somatische Seite der Pflegebedürftigkeit legt und so den steigenden Prävalenzdaten z. B. von Menschen mit demenziellen Beeinträchtigungen nicht gerecht wird.

Im Zusammenhang der Diskussion um das SGB IX und später der Altenberichte der Bundesregierung ist zudem deutlich geworden, wie sehr die in den Einzellogiken der jeweiligen Sozialgesetzbücher zu Tage tretende Leistungsstruktur eine integrative, Teilhabemöglichkeiten stärkende Versorgungsformen nicht stärkt (6. Altenbericht), und zudem durch zersplitterte Beratungsmöglichkeiten und fehlende Transparenz der Angebote die Potentiale der älteren Menschen selbst, die auf eine kontinuierliche, vernetzte Langzeitversorgung angewiesen sind, nicht ausreichend in ihrer Heterogenität wahrnimmt. Besonders bedenklich ist der seit der Einführung der Pflegeversicherung zu beobachtende Prozess einer zunehmenden „Entpflichtung und Selbstentpflichtung der Kommunen, der durch eine mangelnde Dynamisierung der Leistungen heute wieder eine ähnliche Höhe der Sozialhilfeabhängigkeit zur Folge hat wie zum Zeitpunkt der Einführung der Pflegeversicherung. Diese Entwicklung ist aus heutiger Sicht ebenso eine Fehlentwicklung wie die unterschiedliche Risikoverteilung zwischen der gesetzlichen sozialen Pflegeversicherung und der privaten Pflegeversicherung.

Dennoch hat sich die Pflegeversicherung als lernendes System erwiesen, was sich sowohl im Blick auf die rechtliche Weiterentwicklung in den Reformen zeigt, aber auch durch die Akzeptanz, die diese jüngste Entwicklung des Sozi- alversicherungssystems in der Bevölkerung genießt. Diese Akzeptanz ist nicht zuletzt auch deutlich geworden in der Diskussion und den einmütigen Empfehlungen des „Beirats zur Überarbeitung des Pflegebedürftigkeitsbegriffs“ ( 2006-2009). In der Folgezeit wurde die Chance für eine von breiten Mehrheiten getragene Entwicklung verpasst. Noch schärfer als in der Vergangenheit treten jetzt die bis dahin „gefühlten“ Versorgungslücken offen zu Tage, für die mit dem Themenreport der Bertelsmann Stiftung (2012) nun umsetzungsrelevante Daten oder modellhafte Strategien für Quartierskonzepte ( Netzwerk SONG oder auf Länder- und Kommunalebene) zwischenzeitlich vorliegen. Sie verdeutlichen Möglichkeiten, die zu tatsächlichen Alternativen ausgebaut werden müssen.

\section{Gute Pflege vor Ort}

Sicherung und Ausbau von Teilhabe, Pflege und Betreuung gehören zu den herausragenden gesellschaftlichen Aufgaben in diesem Jahrhundert. Wie wir alle wissen, wird sich der Anteil der hilfsbedürftigen Älteren im Vergleich zur Zahl und Anteil Jüngeren sprunghaft erhöhen und die Zahl der älteren Menschen mit körperlichen wie geistigen Einschränkungen zunehmen. Darüber besteht Einvernehmen zwischen

\section{Sicherung und Ausbau von Teilhabe, Pflege und Betreuung gehören zu den herausragenden gesellschaftlichen Aufgaben in diesem Jahrhundert.}

allen Handlungsebenen der Politik und Zivilgesellschaft, Experten und Angehörigen. Trotzdem kann die Pflege trotz einer allseits betonten demografischen Herausforderung nicht mit der nötigen Entschlossenheit und Tatkraft in Bund, Ländern und Gemeinden und anderen Verantwortungsebenen z. B. den Unternehmen rechnen. Trotz verschiedener Reformgesetze wurden überzeugende, durchgreifende Verbesserungen nicht erreicht. Bisher

1. steht ein grundsätzlicher Wandel durch die Einführung des Neuen Pflegebedürftigkeitsbegriffs aus,
2. bleibt die Teilhabemöglichkeit älterer Menschen eine unrealisierte Forderung und

3. werden Lösungen für die demographische Herausforderung, Grundsatzfragen der Infrastruktur und die menschennahe Bündelung von Beratungs- Entscheidungs- und Versorgungsstrukturen nicht durchgreifend angegangen.

4. fehlt nach wie vor ein Gesamtkonzept für Teilhabe, Pflege und Betreuung, dass schon bei der Beauftragung des ersten Beirats für die Überarbeitung des Pflegebedürftigkeitsbegriffs durch das Bundesgesundheitsministeriums (2006) angemahnt worden war.

Das Kuratorium Deutsche Altershilfe hat mit wachsender Enttäuschung und Ernüchterung die fehlende Fähigkeit und Bereitschaft des BMG, die Ergebnisse des Beirats umzusetzen und zu einem wirksamen Strukturwandel zu nutzen, besonders die fehlende Festlegung hinsichtlich des Finanzvolumens und die Unklarheit hinsichtlich des Einführungszeitpunkts des erarbeiteten Pflegebegriffs und des Begutachtungsverfahrens aber auch die Rücknahme bereits erreichter Fortschritte wie den Ansatz der Pflegestützpunkte.

Das Kuratorium Deutsche Altershilfe und die Friedrich Ebertstiftung haben daher 2012 eine gemeinsame Arbeitsgruppe aus Experten unterschiedlicher Profession und Erfahrungsbereiche eingerichtet mit der Bitte, ein Modell für ein zukunftsfähiges Konzept für „Gute Pflege vor Ort. Vom Recht auf eigenständiges Leben im Alter" (Redaktion: D. Engelmann, J. Gohde, G. Künzel, S. Schmidt) $\mathrm{zu}$ beschreiben. Dieses Konzept ist im Juli 2013 als Manuskript vorgelegt worden (Publikation im August in der Reihe „WISO Diskurs“). An ihm orientiert sich die weitere Darstellung.

Die Arbeitsgruppe ist der Überzeugung, dass sich die Entwicklung prinzipiell beherrschen lässt, wenn zeitnah ein Gesamtkonzept umgesetzt wird, das auf Selbstbestimmung setzt, zivilgesellschaftliche und professionelle Pflege vor Ort bündelt, Versorgungsausgaben zielgenau einsetzt und die Gesundheit der Bevölkerung durch Rehabilitation und 
Prävention fördert. Es besteht jetzt die Chance einen grundlegenden Strukturwandel zu gestalten.

Die Politik ist in der Pflicht, ein Gesamtkonzept von Teilhabe, Betreuung, Pflege und Gesundheitsversorgung für alle älteren Menschen anzugehen:

- Die Aufrechterhaltung der Selbstständigkeit alter Menschen und die Achtung ihrer personellen Würde und Selbstbestimmung sind der Kern eines neuen Leitbildes

- Das Recht des alten Menschen auf ein barrierefreies Umfeld in seinem Sozialraum -ob im städtischen Quartier oder im ländlichen Raum- und darauf, auch bei Pflegebedarf möglichst lange in der vertrauten, heimatlichen Umgebung zu bleiben, muss durchgesetzt werden.

- Die Teilhabe der Pflegebedürftigen am öffentlichen Leben und Zugang zu allem, was Lebensqualität ausmacht, ist zu realisieren.

- Gleiche Rechte und gleiche Leistungen für Menschen mit körperlichen und geistigen Einschränkungen müssen in der Pflegeversicherung verwirklicht werden.

Die Arbeitsgruppe rät den gesetzgebenden Körperschaften, zu Beginn der neuen Legislaturperiode eine große Pflege- und Teilhabereform anzugehen, und mit allen Akteuren über die verschiedenen Verantwortungsebenen hinweg einen Aktionsplan mit gemeinsamen sektorenübergreifenden Zielen, einem neuen Leitbild und tragfähigen Lösungen vorzulegen. Die Bedeutung der Aufgabe ist mit dem zu vergleichen, was zur Förderung von Vereinbarkeit von Familie und Beruf und zur Förderung der schulischen Bildung zurzeit verwirklicht wird.“

\section{Voraussetzungen und Annahmen}

Alle Menschen mit Pflegebedarf haben ein Recht auf die Achtung ihrer Würde. Der in der UN-Behindertenkonvention (2009) garantierte Anspruch auf Inklusion gilt auch für alte und pflegebedürftige Menschen.

Pflegebedürftigkeit und Pflege gehören zu einer Gesellschaft des langen Lebens und stehen im Zusammenhang mit dem Wunsch nach Selbstbestimmung, Selbstständigkeit und Teilnahme am gesellschaftlichen Leben in der selbst gewählten Umgebung. Pflege ist der Aspekt des Lebens, an dem sich die Solidarität und Humanität unserer Gesellschaft im Umgang mit hilfsbedürftigen Menschen -alten wie jungen, gesunden und kranken, behinderten und nicht behinderten- beweist.

Langes Leben und die Zunahme des Anteils Älterer an der Bevölkerung bedeuten, dass wir uns als Gesellschaft um eine steigende Zahl Hilfsbedürftiger zu kümmern haben. Der demographische Wandel lässt einerseits den Anstieg pflegebedürftiger Personen sowie andererseits den Rückgang familialer Ressourcen bei gleichzeitigem Schrumpfen der Erwerbsbevölkerung erwarten. In

\section{Die Aufrechterhaltung der Selbstständigkeit und die Achtung der personellen Würde und Selbstbestimmung müssen auf allen Ebenen durchgesetzt werden.}

spektive. Ein Ausbau der professionellen Angebote, Kleinräumigkeit und gelebte Solidarität im Sozialraum und der gezielte Ausbau professioneller Angebote sind die Forderungen für die Zukunft, um die Würde der Menschen bis ins hohe Alter zu garantieren. Es bedarf einer Wiederentdeckung der Rolle der Kommunen.

Die Gesellschaft wird bunter und vielfältiger. In Zukunft steigt die Zahl pflegebedürftiger Menschen mit Migrationshintergrund. Fachliche Standards, Ausbildung und die Gesellschaft müssen sich kultur- sensibel darauf einstellen.

Wenn fortschreitender Hilfebedarf das selbstständige Leben mehr und mehr einschränkt, sind Menschen mehr und mehr auf Unterstützung und auf eine gute Pflege angewiesen. Pflegepolitik muss mehr als Pflegeversicherungspolitik sein. Sie muss Prävention, Rehabilitation, Teilhabe, optimierte medizinische Versorgung sowie den Bedarf an Dienstleistungen für Altershaushalte im Vorfeld der Pflegebedürftigkeit zum Gegenstand haben. Sie muss

der Zukunft kann nicht mehr selbstverständlich davon ausgegangen werden, dass weibliche Familienangehörige die Pflege übernehmen.

Kognitive und psychische Beeinträchtigungen, vor allem die Altersdemenz, werden neben körperlichen Beeinträchtigungen Merkmal von Pflegebedürftigkeit. Mit steigender Multimorbidität steigt die Zahl der Pflegebedürftigen und macht eine nahtlose Zusammenarbeit pflegerischer, alltagsrelevanter und gesundheitlicher Hilfen erforderlich.

Pflege betrifft alle: Es geht um ein Gerechtigkeitsthema aller Generationen. Die notwendige Hilfe darf nicht von den Einkommens- und Vermögensverhältnissen abhängen, die Hilfeleistung muss auch bei ungünstigen ökonomischen Ausgangslagen gesichert werden.

Die Gesellschaft unterliegt einem tiefgreifenden Wandel in der Lebenskultur und in der sozialen Struktur. Die heutigen Versorgungsarrangements stehen jetzt und mehr noch in Zukunft nicht mehr in Übereinstimmung mit den sich wandelnden Einstellungen und Bedürfnissen der Menschen. Immer mehr und größere Heime sind keine Zukunftsper- aus dem sozialrechtlichen Kostendenken heraustreten und die wirtschaftlichen Potenziale dieses Sektors mitdenken. Die Koordinationsmängel zwischen den verschiedenen Entscheidungs- und Leistungsträgern, die daraus resultierenden Unter- und Überversorgungen und die im Einzelfall an Hilfeverweigerung grenzenden Verzögerungen der Leistungsgewährung sind nicht länger hinnehmbar.

\section{Neues Leitbild für eine teilhabeorientierte Pflege vor Ort in verlässlicher Solidarität}

Die demographischen, gesundheitlichen, sozialen, personellen und ökonomischen Herausforderungen erfordern ein neues Leitbild.

Die längst mögliche Aufrechterhaltung der Selbstständigkeit und die Achtung der personellen Würde und Selbstbestimmung sind der Kern des pflegerischen Leitbildes, das sektorenübergreifend auf allen Ebenen der Verantwortung und Zuständigkeit durchgesetzt werden muss.

Pflege muss unterschiedliche Lebenslagen berücksichtigen und von der 
Gleichwertigkeit von psychisch-kognitiven und somatischen Beeinträchtigungen der Selbstständigkeit eines Menschen mit Pflegebedarf ausgehen. Vorrangig sind inklusive Hilfen, haushaltsnahe Dienstleistungen, Hilfen zur Teilhabe am Leben in der Gemeinschaft

\section{Die Zukunft der Pflege liegt im städtischen Quartier und in der dörflichen Gemeinschaft.}

bis hin zu den gesundheitlichen Hilfen für die multimorbiden schwer Pflegebedürftigen, Hilfen für Menschen mit Demenz und andere psychisch beeinträchtigte Menschen in ihren angemessenen Versorgungsformen.

Rehabilitation und Prävention haben Vorrang vor Pflege. Pflegebedürftigkeit muss möglichst lange vermieden werden. Eine Weiterentwicklung der Kooperation und eine bessere Vernetzung der Haus- und Fachärzte mit ambulanten Pflegediensten, den stationären medizinischen und pflegerischen Einrichtungen sowie der Palliativversorgung sind notwendig.

Das neue Leitbild ist ein Plädoyer für selbstbestimmtes Leben in verlässlicher Solidarität und gleichzeitig Subsidiarität, für die Absicherung durch die Sozialversicherungssysteme und durch eine neue Kultur des Helfens in der unmittelbaren Lebensumgebung der älteren Menschen.

Pflege ist vor Ort neu zu denken, die kommunale und regionale Ebene ist dazu zu stärken. Eine wohnortnahe, personenorientierte, beteiligungsorientierte Versorgung lässt sich nur im örtlichen Verbund organisieren. Die Zukunft der Pflege liegt im städtischen Quartier und in der dörflichen Gemeinschaft. Ziel ist der möglichst lange Verbleib in der häuslichen Umgebung mit Hilfe von verlässlichen sozialen Netzwerken

Das neue Leitbild bezieht sich auch darauf, dass in Zukunft sowohl Männer als auch Frauen in die familiale, ehrenamtliche und professionelle Pflege einbezogen sind. Es setzt voraus, dass Männer wie Frauen erwerbstätig sind, sich aber gleichzeitig auch um hilfebedürftige Angehörige kümmern und Pflegearbeiten leisten.

Assistenz orientiert sich passgenau am Prinzip der Hilfe zur Selbsthilfe und der Unterstützung zum frühestmöglichen Zeitpunkt. Das erfordert Begleitung von Angehörigen und Menschen mit Pflegebedarf sowie lokale Arrangements von Unterstützung und Beratung, neue flexible Dienstleistungsformen, den Ausbau von barrierefreien Wohnungen und Wohnumgebungen für alle Generationen, eine gut vernetzte medizinisch-pflegerische qualitätsgesicherte Unterstützung, personbezogene technische Hilfen und die Entwicklung des Sozialraums. Die stationären Einrichtungen gehören mit neuen Aufgaben eines Netzwerkzentrums und als Versorgungsform für ambulant nicht mehr auffangbare Situationen dazu.

Für die unterschiedlichen Kompetenzen in den medizinisch-pflegerischen, sorgenden, sozialarbeiterischen, Alltagsgestaltenden und hauswirtschaftlichen Bereichen wird eine zunehmende Zahl von Fachkräften benötigt, welche die Qualität der Versorgung und die Unterstützung und Entlastung der $\mathrm{Fa}$ milienangehörigen gewährleisten und die Voraussetzungen für das Zusammenwirken mit der Zivilgesellschaft bei der Erfüllung der Aufgaben schaffen. Die Pflegearbeit insgesamt bedarf der Aufwertung, sei sie privat, ehrenamtlich oder professionell geleistet. Dazu gehören Regelungen zur Vereinbarkeit von Pflege und Erwerbstätigkeit für Männer und Frauen, Initiativen zur Förderung des ehrenamtlichen Engagements und die Aufwertung der professionellen Arbeit. Der Pflegesektor ist weniger als Last und mehr als Beschäftigungssektor mit hohem ökonomischem Potenzial zu sehen. Deutschland hat einen Nachholbedarf im Ausbau personenbezogener Dienstleistungen, auch im Vorfeld der Pflege.

\section{Welche Schwachstellen müssen bearbeitet werden:}

In knapp 20 Jahre alte Pflegeversicherung hat sich eine wettbewerbsorientierte Versorgungslandschaft staatlicher, gemeinnütziger, kirchlicher und privater Pflegeanbieter entwickelt, die große Fortschritte für die Pflegebedürftigen gebracht hat.
Es sind aber auch Schwachstellen deutlich geworden, die heute Veränderungen erfordern.

\section{Pflege ist mehr als \\ Pflegeversicherung und mehr als Minutenpflege.}

Die Entwicklung des SGB XI hat ein "Produktdenken“ und eine Kommerzialisierung in der Pflege bewirkt, deren schädliche Auswirkungen in der gehetzten Minutenpflege, fehlender Beziehungspflege und hoch belastetem Personal sichtbar werden. Wettbewerbsund Konkurrenzfähigkeitskriterien, die Orientierung auf den niedrigsten Preis in den Vergütungsverhandlungen und die lange ausgebliebene Dynamisierung der Leistungsbeträge befördern den Rationalisierungsdruck. Der mit dem Teilleistungssystem verbundene Finanzdeckel fördert zusammen mit der auf dem Verrichtungsbezug beruhenden Praxis eine Wahrnehmung der Rationalisierbarkeit der Pflege. Diese Sicht lässt die Beziehungsorientierung der Pflege außer Acht und würdigt zu wenig, dass Pflegequalität auch zugewendete Zeit beinhaltet und deshalb einer anderen Logik folgt als die Arbeit zur Produktion von

\section{Care ist ein zentrales Element sozialer Kohäsion und damit gesellschaftlicher Vorsorge.}

Gütern. Kurz: Zeitrationierung bedeutet Qualitätsminderung. Minutenpflege wird besonders der zunehmenden Zahl von Menschen mit psychisch-kognitiven Beeinträchtigungen ihrer Alltagskompetenz und ihren sozialen Bedarfen nicht annähernd gerecht. Auch die höheren finanziellen Unterstützungsleistungen, die das Pflege- Neuausrichtungsgesetz für die Pflegebedürftigen und ihre Angehörigen gebracht hat, können eine veränderte Zugangsgestaltung nicht ersetzen, weil ohne sie keine Gleichwertigkeit von Beeinträchtigungen erreichbar ist.

Die Entpflichtung und Selbstentpflichtung der Kommunen war eine Fehlentwicklung. Care ist ein zentrales Element sozialer Kohäsion und damit gesellschaftlicher Vorsorge. Zuständigkeiten und Verantwortung müssen dem entsprechen und das Leistungsrecht muss dies abbilden. Dies ist bislang nicht der Fall. 


\section{Fehlende Careorientierung}

Care (Sorge) Arbeit wird gegenwärtig weitgehend im privaten Raum geleistet. Über die Belastungen, Erkrankungen und die psychische Gesundheit der pflegenden Familienangehörigen ist wenig bekannt. Ihre Arbeitsleistung wird unzureichend gewürdigt. In vielen Fällen führt die private Pflege für diejenigen, die sie leisten (vor allem für Frauen) in die spätere Armut und eine vorzeitige eigene Pflegebedürftigkeit. Darüber hinaus fehlt es an einer gezielten Aktivierung der bürgerschaftlichen Ressourcen und an zivilgesellschaftlichen Beteiligungsmöglichkeiten bei der Gestaltung der Begleitungs- und Pflegeangebote. Für dieses wichtige Aufgabenfeld muss es eine gesetzlich geregelte Verantwortung geben. Careorientierung, die eine Konzentration auf den Zusammenhalt des Sozialen und neue Vorsorge ermöglicht, konnte so nicht entstehen. Hierfür ist die Aktivierung von vorhandenen Ressourcen durch Rehabilitation und Prävention in Zuständigkeit der Pflege erforderlich, wie die Würdigung und Einbeziehung von Netzwerken aus Nachbarn und Freunden.

\section{Anreize}

Die gegenwärtigen Markt- und Systemanreize im Pflegesystem wirken nicht auf den Ausbau integrierter Versorgungsstrukturen hin und berücksichtigen unzureichend den Wunsch der Menschen nach selbstständiger Lebensgestaltung. Fehlanreize gehen von der unterschiedlichen Finanzierung stationärer und ambulanter Versorgungsformen im Leistungs- und Vergütungsrecht der Pflegeversicherung und von der Behandlungspflege in Heimen durch das SGB XI aus.

\section{Entpflichtung und Selbstentpflichtung der Kommunen}

Die durch einen Prozess der „Entpflichtung und Selbstentpflichtung der Kommunen" nach Einführung der Pflegeversicherung im Jahr 1995 defizitäre Infrastruktur im Umfeld, insbesondere die ungenügende Unterstützung des selbstbestimmten Wohnens und Lebens im Alter, verstärkt diese Fehlsteuerung. Demgegenüber stehen die Wünsche und Bedarfe der pflegebedürftigen Menschen. Der Vorrang der Auswahlentscheidung der Betroffenen wird durch diese Angebotssteuerung eingeschränkt und Abhängigkeiten können entstehen. Die Maßnahmen und Strukturen, die korrigierend wirken können, wie Beratung und Case Management (individuelle Fallbegleitung), die Ausrichtung und die finanziellen Anreize des Leistungs- und Vergütungsrechts, kommunale Infrastrukturpolitik, die örtliche Koordinierung oder die Durchsetzung von Rechtsansprüchen sowie Qualitätssicherung wirken zum Teil in unterschiedliche Richtungen und sind nicht wohnortnah und verbindlich auf das Ziel des selbstbestimmten Lebens und Wohnens mit Unterstützungsbedarf abgestimmt. Die Sicherstellung der Leistungen ist (unbeschadet der Ergänzungsfunktion der Sozialhilfe) eine vorrangige Aufgabe der Sozialversicherung. Die Koordinierung der örtlichen Akteure (Kostenträger, Leistungserbringer und Zivilgesellschaft) und der Dienstleistungs- und Beratungsstruktur, an der beide mitwirken müssen, erfordert aber wegen des örtlichen Bezugs eine kommunale Federführung.

Äußerungen und Positionspapiere des Deutschen Vereins und der Kommunalen Spitzenverbände lassen in den letzten Jahren ein wachsendes Interesse an den Aufgaben öffentlicher Aufgaben in der Altenhilfe erkennen.

\section{Wir sind weit davon entfernt den Bereich der Teilhabe als eine zentrale Zukunftsinvestition anzugehen.}

Institutionelle Eigeninteressen, die Sektorierung von Leistungen, unterstützt von fehlenden integrativen Versorgungsmodellen, Fehlanreizen und ungeklärten Schnittstellen zwischen Leistungsgesetzen, dominieren bei den Verantwortlichen für das Leistungsgeschehen.

Wir sind weit davon entfernt den Bereich von Teilhabe als eine zentrale Zukunftsinvestition anzugehen und die Unterbewertung der Arbeit der Fachkräfte und der Sorgearbeit der Angehörigen aufzuheben.

\section{Gute Arbeit}

In der Pflege arbeiten heute Hunderttausende Menschen. Ihr Arbeitsalltag ist dadurch geprägt, dass sie überwiegend sehr hart arbeiten. Ihr Ansehen in der Öffentlichkeit entspricht nicht ihrer Leistung.

Der Arbeitsmarkt muss die strukturellen Veränderungen in der pflegerischen und sozialen Versorgung einbeziehen. In erster Linie muss der heimische Arbeitsmarkt für die Pflege ergiebig sein. Wir werden aber ohne MigrantInnen nicht auskommen.

Die Fachkräfte werden mit hohen Anforderungen an die Qualifikation konfrontiert- am besten auf europäischem Niveau. Verbesserte Ausbildungs- und Arbeitsbedingungen, neue Berufe in Pflege und Begleitung, und neue Anforderungen an die Fachkräfte (z.B. Koordination und Qualitätssicherung, Kooperation mit Pflegenden mit Migrationshintergrund) erfordern neue Strategien auch von den Arbeitsagenturen.

\section{Anforderungen und Chancen für eine Reform}

Die Sicherung und Entwicklung der Pflege lässt sich durch eine Verbesserung der Infrastruktur von Wohnen und Teilhabe vor Ort, der Verantwortlichkeiten und Versorgungsstrukturen unter Beteiligung der Betroffenen modernisieren. Die Zukunft von Teilhabe, Pflege und Assistenz entscheidet sich heute. Sie muss vor Ort neu gedacht und gestaltet werden.

1. Der neue Pflegebedürftigkeitsbegriff muss zügig eingeführt werden. Mit ihm und mit dem neuen Begutachtungssystem ist ein Weg beschrieben, der die Realisierung eines Gesamtkonzepts ermöglicht und die Gestaltungsräume für neue angemessene Leistungen schafft. Das heutige Minuten-Diktat entfällt. Die Chancen, die der neue Begriff für die Gestaltung neuer rehabilitativer und präventiver Veränderungen in Leistungen und Versorgungsstrukturen und die Kernprozesse der Pflege bietet, müssen genutzt werden.

2. Das Teilleistungssystem der Pflegeversicherung muss finanziell gestärkt werden. Die Leistungen müs- 
sen durch Dynamisierung vor ihrer Aushöhlung geschützt werden.

3. Der neue Pflegebegriff muss übergreifend im SGB I verortet werden. Die Pflegekassen werden Rehabilitationsträger für die von Ihnen zu erbringenden Pflegeleistungen. Die verbindliche Kooperation der für Leistungen und Infrastruktur Verantwortlichen ist gesetzlich zu regeln. Die Bearbeitung von Schnittstellen und Kostenverschiebungen darf kein Tabu mehr sein. Die Koordination dieser verbindlichen $\mathrm{Zu}$ sammenarbeit auf örtlicher Ebene ist Aufgabe der Kommunen und Länder.

4. Die notwendige Sozialraumorientierung der Pflege erfordert, dass die Kommunen eine stärkere, die Infrastruktur steuernde Rolle übernehmen. Das erfordert ein Umdenken und eine ausreichende Finanzausstattung. Es müssen neue Wohnformen ausreichend gefördert werden. Wir brauchen ein Investitionsprogramm „Pflege und Wohnen“.

5. Der Grundsatz „ambulant vor stationär" muss konsequent umgesetzt werden. Die Zukunft der Pflege liegt im Quartier. Für die bestehenden ambulanten und stationären Einrichtungen ergeben sich durch Vernetzung von Angeboten gute Chancen im Modernisierungsprozess. Es muss selbstverständlich werden Schnittstellen zu vermeiden, Verantwortung zu teilen, Nachbarn und Freunde im Rahmen von Assistenzmodellen zu beteiligen und einen „Hilfemix“ zu gestalten.

6. Der Ausbau von bezahlbaren haushaltsnahen Dienstleistungen ist zur Unterstützung und zum Erhalt der Selbstständigkeit und damit zur Verzögerung von Pflegebedürftigkeit unerlässlich.

7. Das Beratungsangebot muss flächendeckend wohnortnah, verbindlich und gemeinsam ausgebaut werden; die vorhandenen Beratungsstrukturen nach $\$ 7$ a SGB XI( Pflegeberatung), \$92c SGB XI (Pflegestützpunkte) und $\$ 23$ SGB XI (Gemeinsame Servicestellen) sind die Pfeiler für neue Strukturen.

8. Die Vereinbarkeit von Pflege und Beruf muss massiv unterstützt und arbeitsrechtlich ausgeweitet werden. Mittelfristig liegt hierin ein großer gesamtwirtschaftlicher Vorteil.

9. Auch in der Personaldiskussion ist ein Paradigmenwechsel erforderlich. Der Weg eines Teilhabeorientierten Konzepts erfordert

- neue Fachkräfte mit unterschiedlichen Qualifikationsprofilen

- eine erhebliche verbesserte Ausbildung, neue Arbeitszeitmodelle und personalwirtschaftliche Strategien,

- mehr Personal in Einrichtungen und Diensten.

- Pflege und Assistenz darf kein prekärer Beschäftigungssektor sein. Mitarbeitende müssen generell nach Tariflohn bezahlt und Geschlechtergerecht eingestuft werden. Qualifizierte Arbeit, attraktive Vergütung und ausreichende Unternehmenserträge gehören zusammen.

\section{Gute Pflege ist möglich}

Erfolg versprechen Reformen in den nächsten Jahren dann, wenn sie keine Entwicklungen verbauen, die zu mehr kommunaler und zivilgesellschaftlicher

Das weitere Hinauszögern von nötigen Entscheidungen wird noch mehr Engpässe schaffen und die Akzeptanz für Dienstleistungen vermindern.

Verantwortung führen und durch den Abbau einseitiger Entwicklungen unnötige, nicht demografiebedingte Kostensteigerungen vermeiden. Dazu zählen Klarstellungen der Zuständigkeiten und Verantwortlichkeiten für die Pflege auf allen Ebenen, in Ausbildungsfragen ebenso wie in der Vermeidung von Versorgungslücken durch intelligente Verknüpfungen in der Gesundheitsversorgung

- durch eine verbesserte Kooperation der Professionen vor Ort,

- durch eine Weiterentwicklung des Leistungsrechts an Schnittstellen, wie am Übergang vom Krankenhaus in die ambulante oder stationäre Versorgung,

- durch ein personalwirtschaftliches Versorgungsmanagement, das heterogene Bedarfe und die Diversität von Personalressourcen ernst nimmt. Die
Stärkung der Pflegenden selbst steht dabei im Vordergrund. Sie dürfen nicht länger als „Stille Helden“ angesehen werden.

Nicht zuletzt wollen die Bürger sich beteiligen an der Lösung von Problemen. Das Thema ist in der Mitte der Bevölkerung „angekommen“. Erkennbar geht es um den Zugang für alle. Das weitere Hinauszögern von nötigen Entscheidungen und die Fortsetzung eines nicht konsequent rehabilitationsorientierten Konzeptes geht schon jetzt erkennbar an den Wünschen und an den Bedarfen der Bürger vorbei und wird künftig bei begrenzten Mitteln noch mehr Engpässe schaffen und die Akzeptanz für Dienstleistungen vermindern. Aufwendungen für eine moderne Infrastruktur wie die Entwicklung haushaltsnaher Dienstleistungen, die ein selbstständiges, gutes Leben im Alter, Wohlbefinden und Partizipation möglich machen, sind jedoch Zukunftsinvestitionen in die regionale Infrastruktur, die große Auswirkungen auf den Wirtschaftsstandort haben, weil sie die Vereinbarkeit von Sorgearbeit und Erwerbsarbeit ermöglichen. 